\title{
CANADLANA
}

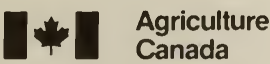

Publication 1499/F

\section{Les mouches noires}

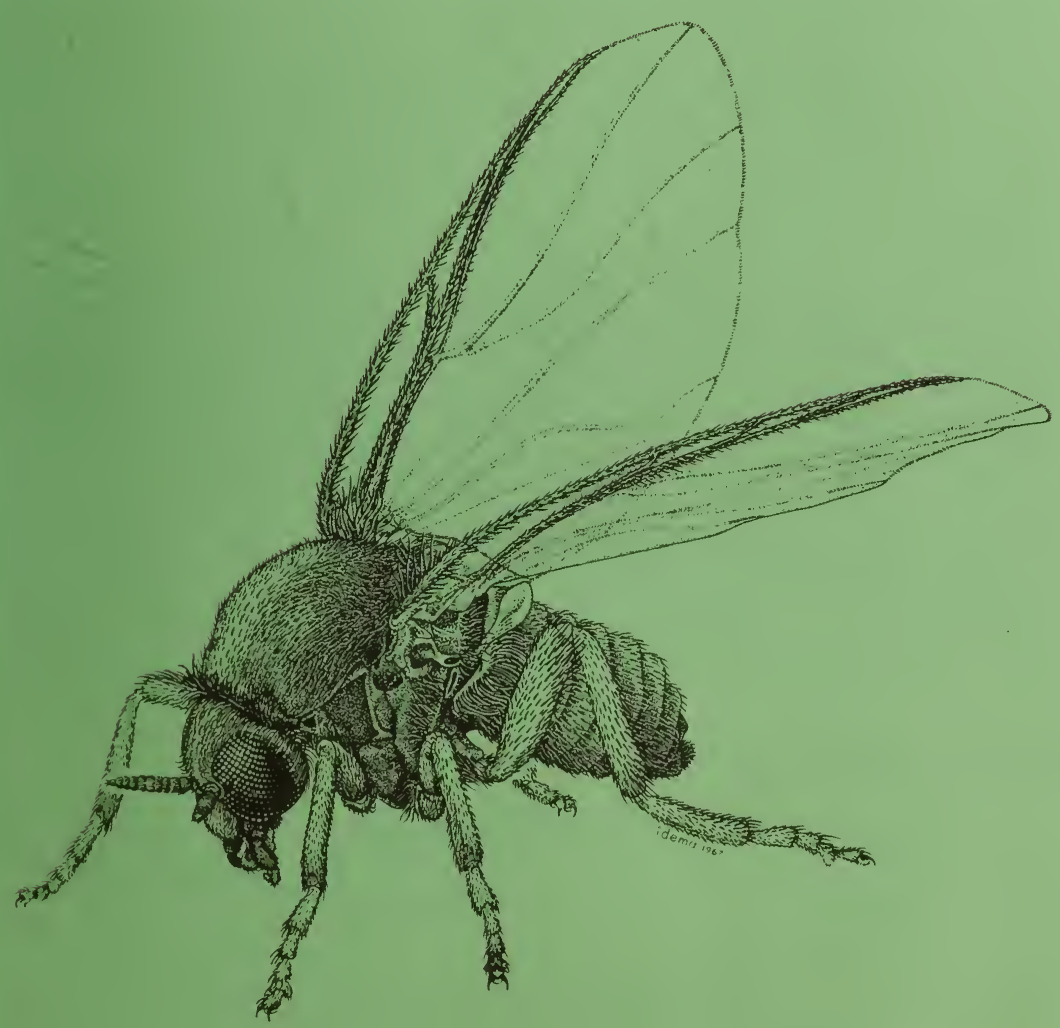

Canadä 
Digitized by the Internet Archive in 2013 


\title{
Les mouches noires
}

\author{
P. G. Mason
}

Station de recherches

Saskatoon, Sask.

J. A. Shemanchuk

Station de recherches

Lethbridge, Alberta

Les recommandations contenues dans cette publication au sujet de l'utilisation des pesticides doivent être considérées comme des directives seulement. Toute application d'un pesticide doit être faite conformément aux instructions imprimées sur l'étiquette de ce pesticide, conformément à la Loi sur les produits antiparasitaires. Il faut toujours lire l'étiquette. En outre, l'utilisation d'un pesticide enregistré doit faire l'objet d'une recommandation des autorités provinciales. Comme ces recommandations peuvent varier d'une province à une autre, vous devriez consulter le représentant du ministère de l'Agriculture de votre province pour obtenir des avis particuliers.

Agriculture Canada Publication 1499/F

On peut en obtenir des exemplaires à la

Direction générale des communications

Agriculture Canada, Ottawa K1A 0C7

- Ministre des Approvisionnements et Services Canada 1990

No de cat. A43-1499/1990F ISBN 0-662-95722-9

Impression 1973 Réimpression 1974 Révision 1990 2M-04:90

Also available in English under the title:

Black flies 



\section{TABLE DES MATIERES}

Introduction 7

Cycle vital 7

Comportement 8

Espèces qui s'attaquent à l'homme 8

Espèces qui s'attaquent au bétail 9

Bovins 9

Chevaux 10

Volaille 11

Moutons 11

Porcs 11

Protection 12

Protection des humains 12

Vêtements 12

Répulsifs 12

Protection du bétail 12

Bovins 13

Chevaux 14

Volaille 14

Moutons et porcs 14

Lutte 14

Planification d'un programme de lutte 14

Évaluation de la gravité de l'attaque 14

Identification des espèces nuisibles et des zones de reproduction 15

Type de programme de lutte 15

Destruction des larves 16

Insecticides disponibles 16

Matériel 16

Calculs des doses de larvicide 17

Épandage 18

Destruction des adultes 18

Insecticides disponibles 19

Matériel 19

Épandage 19

Évaluations et rapports 20 


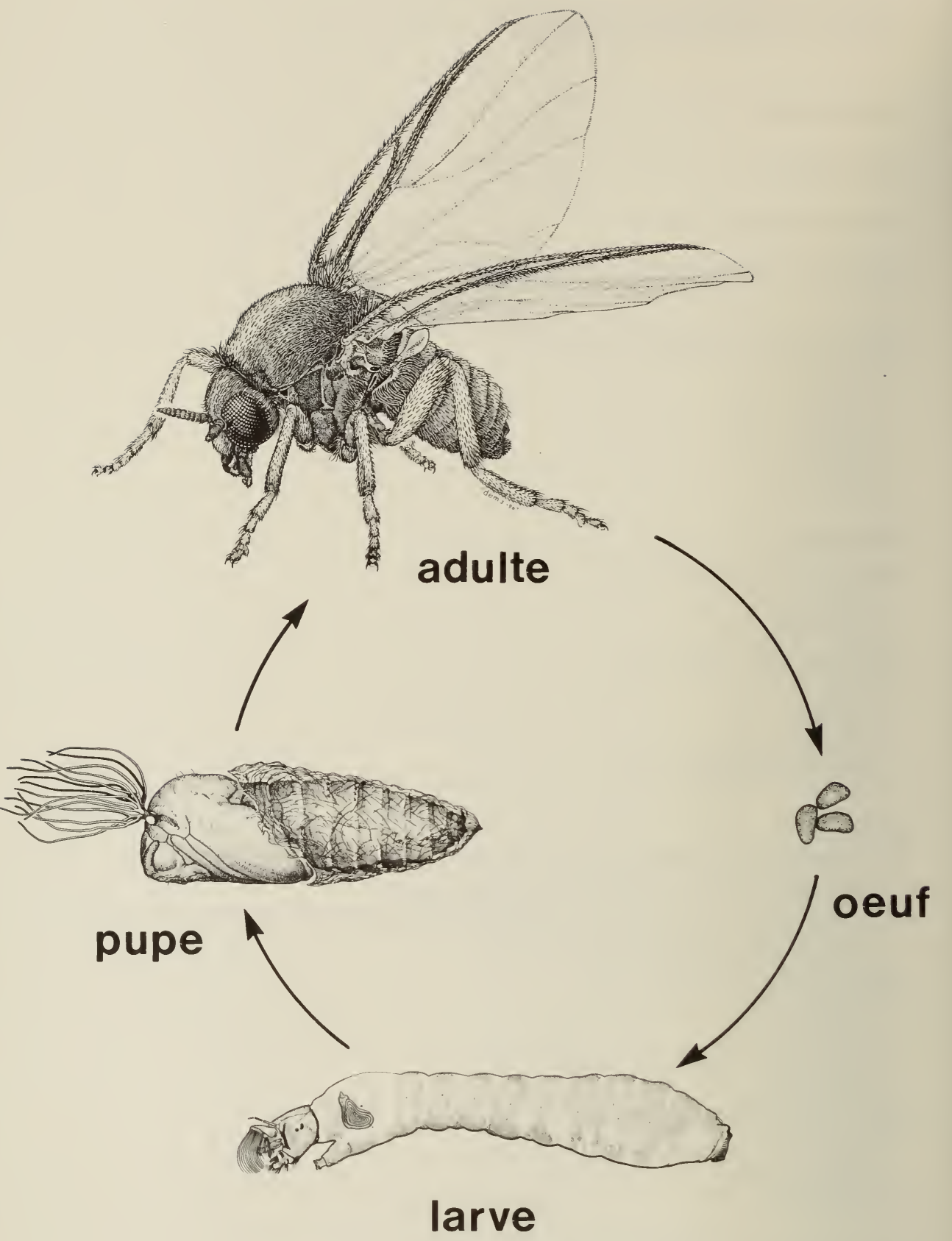

Fig. 1 Cycle biologique des mouches noires. (Illustrations de la larve, de la pupe et de l'adulte, reproduites avec l'autorisation du ministère des Approvisionnements et Services Canada et inspirées du Manual of Nearctic Diptera, Volume 1, de McAlpine et coll., 1981.) 


\section{INTRODUCTION}

Les mouches noires, dont il existe environ 100 espèces au Canada, se retrouvent en plus ou moins grande quantité dans toutes les régions du pays. Les adultes femelles s'alimentent de sang, ce qui a pour effet d'irriter et d'incommoder les humains, les mammifères domestiques et sauvages, et les oiseaux. Outre les ennuis et les malaises qu'elies provoquent, les mouches noires causent des pertes économiques qui se traduisent par un fléchissement de la production laitière et bovine, une baisse de l'efficacité des travailleurs agricoles et industriels, des inconvénients pour les activités de loisirs, une diminution de la valeur des biens immobiliers, la propagation des maladies et la perturbation des opérations militaires. Au Canada, les mouches noires transmettent des parasites du système sanguin aux dindons, aux oies et aux canards.

Les piqûres des mouches noires sont douloureuses et provoquent souvent chez les humains des réactions allergiques qui nécessitent des soins médicaux. Ces réactions peuvent entraîner la mort chez les bovins. De grandes quantités d'adultes femelles du Simulium arcticum Malloch, qui proviennent de la rivière Athabasca, et du $S$. luggeri Nicholson \& Mickel de la rivière Saskatchewan ont tué des bovins et sont considérées comme un facteur qui limite la production de bovins dans ces régions.

Comme les mouches noires se reproduisent dans les eaux courantes des rivières, des ruisseaux et des canaux d'irrigation, elles abondent d'ordinaire tout au long du printemps, de l'été et au début de l'automne, indépendamment des précipitations. Dans certaines régions irriguées, les mouches noires sont suffisamment abondantes pour perturber la vie des humains et des animaux.

La réussite d'un programme de lutte contre les mouches noires exige une planification soigneuse. Les plans devront tenir compte des espèces présentes, de l'emplacement de leurs zones de reproduction, de leur comportement selon les conditions météorologiques et de leurs habitudes lorsqu'elles sont en quête d'hôtes.

\section{CYCLE VITAL}

La croissance des mouches noires comporte quatre stades : oeuf, larve, pupe, adulte (fig. 1). On ne rencontre les trois premiers stades que dans les rivières et les cours d'eau. Certaines espèces pondent des oeufs en masses sur les plantes et les rochers partiellement submergés; d'autres les éparpillent sur la surface d'une rivière. L'éclosion se produit en quelques jours ou en quelques mois, suivant les espèces. Ensuite les larves sont emportées par le courant et se fixent aux rochers et à la végétation dans les endroits où le courant est le plus rapide. Les larves, qui sont d'un gris verdâtre ou brun, 
s'alimentent en filtrant les éléments nutritifs de l'eau et, avec les températures de l'été, elles grandissent jusqu'à atteindre au moins $6 \mathrm{~mm}$. Elles se métamorphosent en nymphes en 2 ou 3 semaines.

Après la nymphose qui ne dure que quelques jours, les adultes sortent de la pupe et flottent à la surface dans une bulle d'air. Les mouches noires adultes, mâles et femelles, se nourrissent du nectar et de la sève des végétaux pour satisfaire leurs besoins énergétiques. L'accouplement se produit peu après l'émergence. Les femelles des espèces piqueuses cherchent alors le sang dont elles ont besoin pour produire des oeufs.

Dans le sud du Canada, les mouches noires font leur apparition en avril, atteignent leur nombre maximal en juin et juillet, puis persistent jusqu'à la première gelée importante d'octobre. Dans les régions plus au nord, la saison débute plus tard et se termine plus tôt. Certaines espèces, comme le $S$.luggeri, produisent plus d'une génération par an et leurs nombres peuvent atteindre des niveaux épidémiques, si rien n'est fait pour les éliminer.

Les femelles de la plupart des espèces de mouches noires ont besoin de sang pour produire des oeufs. Dès qu'elles repèrent un hôte, elles se posent sur sa peau, $y$ insèrent leurs organes buccaux tranchants, puis se gorgent de sang. Une fois rassasiées, elles atteignent d'ordinaire deux fois leur taille normale.

\section{COMPORTEMENT}

Les mouches noires se manifestent pendant la journée, maais leurs attaques sont plus nombreuses le matin et l'après-midi. Lorsque le temps est nuageux ou que l'orage menace, elles attaquent brutalement à tout moment du jour. Les vents peuvent entraîner de fortes quantités d'adultes des zones de reproduction vers les régions avoisinantes, particulièrement les terres agricoles. Un changement de la direction du vent peut entraîner les mouches noires vers d'autres régions. Le froid ou la pluie en général arrête momentanément une infestation.

\section{ESPÈCES QUI S'ATTAQUENT AUX HUMAINS}

Le S. venustum Say, le S. verecundum Stone \& Jamnback, le S. luggeri, le S. decorum Walker, le Prosimulium mixtum Syme \& Davies et le $P$. hirtipes (Fries) sont les principales espèces de parasites qui s'attaquent aux humains au Canada. Ces espèces, dont la couleur varie de brun foncé à noir, peuvent se manifester n'importe quand durant le printemps et l'été. Les attaques peuvent se produire à de nombreux kilomètres des zones de multiplication. Contrairement aux moustiques, qui attaquent habituellement vers la fin du jour et au 
début de la matinée, les mouches noires attaquent leurs hôtes tout au long du jour, mais rarement à l'intérieur.

Le S. vittatum Zetterstedt a une grosse taille et est de couleur grise. It est également attirée par les humains et pique parfois. Cette espèce constitue davantage une gêne qu'une espèce nuisible, parce qu'elle tourbillonne autour de la tête. Le S. griseum Coquillett, le $S$. bivittatum Malloch, le $S$. tuberosum Lundstrom et le $S$. parnassum Malloch sont des parasites qui s'attaquent de temps à autre aux humains, particulièrement dans les régions proches des ruisseaux.

Les femelles des mouches noires sont attirées par les vêtements foncés. Elles piquent les parties exposées du corps, comme la nuque, la naissance des cheveux autour du front et des tempes, le visage, les poignets, le dos de la main et les chevilles. Lorsque les populations de mouches noires sont élevées, ces dernières s'introduisent dans les oreilles et le nez et autour des yeux. Elles lacèrent la peau et y injectent un puissant anticoagulant provenant de leur salive afin d'empêcher la coagulation du sang dans leurs organes buccaux. L'anticoagulant provoque également l'écoulement du sang pendant quelque temps, après que la femelle a quitté son hôte. Une lésion se forme d'ordinaire dans la région piquée, suivie d'un exsudat, d'un oedème et d'une démangeaison. La rougeur et l'oedème peuvent persister jusqu'à $72 \mathrm{~h}$ et la démangeaison jusqu'à 1 semaine. Les piqûres de mouches noires peuvent provoquer des réactions comme une tuméfaction locale, le gonflement des ganglions lymphatiques et des infections secondaires, lorsqu'on gratte la région piquée. Les enfants en général risquent davantage d'être attaqués par les mouches noires que les adultes.

\section{ESPÈCES QUI S'ATTAQUENT AU BÉTAIL}

\section{BOVINS}

Sur le plan économique, environ 11 espèces de mouches noires présentent une importance en tant que parasites des bovins. Le $S$. arcticum et le S. luggeri en Alberta et en Saskatchewan et le $S$. defoliarti Stone \& Peterson dans certaines vallées des régions montagneuses de la Colombie-Britannique s'attaquent sournoisement aux bovins et leurs piqûres causent parfois la mort. Les bovins sont d'ordinaire piqués au ventre, au pis et, dans une moindre mesure, aux pattes, sur les parties latérales du corps, chaque côté du cou, au visage et, lorsque les populations de mouches sont très denses, autour des yeux. Les bovins réagissent contre ces attaques en agitant vigoureusement leur queue, en tapant du pied, en donnant des coups d'oreilles ou en se contractant la peau et en se léchant constamment le corps. Lors d'attaques sérieuses, les bovins cherchent à s'abriter dans les étables ou les broussailles ou se dirigent vers des endroits élevés et 
exposés au vent en terrain découvert, où ils se tiennent debout ou se couchent en groupes serrés.

Le $S$. arcticum est d'ordinaire un parasite des bovins du début de juin à la mi-septembre. Les femelles s'attaquent aux bovins le jour : elles leur font subir des piqûres douloureuses, sucent leur sang et leur injectent une salive toxique. La salive provoque des symptômes analogues au choc anaphylactique (allergique) et, lorsque de grandes quantités de mouches noires piquent des veaux nouveau-nés et d'autres bovins qui n'ont jamais été piqués auparavant, l'anaphylaxie entraîne généralement la mort. L'éleveur subit d'autres pertes par suite de la réduction du pâturage, de la non-fécondation des vaches et de la stérilité possible des taureaux.

Le $S$. luggeri est une espèce qui se manifeste d'ordinaire du début de juin au début d'octobre. Comme la plupart des autres espèces, les femelles attaquent les bovins durant le jour en leur infligeant des piqûres douloureuses et en suçant leur sang. On ne croit pas que le $S$. luggeri cause la mort chez les bovins par injection de salive toxique. Toutefois, en réaction aux essaims compacts, les bovins s'agitent, se pressent contre les clôtures ou même s'affolent. Les pertes que subit le producteur découlent de la réduction du pâturage, de l'interruption de la reproduction, des lésions et des décès causés par le rassemblement des bovins en groupe serré et par leur fuite précipitée. Les veaux nouveau-nés peuvent mourir de déperdition sanguine. Comme les femelles du S. luggeri piquent autour des yeux, on les soupçonne de transmettre la kératite bovine.

Le $S$. defoliarti se manifeste en Colombie-Britannique en juillet et perturbe, durant certaines années, la vie des bovins. Les femelles attaquent d'une manière très semblable à celle décrite pour les femelles du S. arcticum, sauf qu'elles n'injectent pas une salive toxique qui peut entraîner la mort.

Le $S$ ? vittatum est l'une des premières espèces qui apparaissent au printemps et persistent jusqu'en été. Les femelles attaquent les oreilles des bovins et peuvent causer des dermatoses graves.

Le $S$. venustum et le $S$. decorum piquent les bovins dans toutes les parties du corps où elles peuvent s'introduire dans le pelage. Ces espèces ne tuent pas les bovins.

Parmi les autres espèces qui attaquent les bovins au Canada, mentionnons le $S$. tuberosum, le $S$. griseum, le $S$. parnassum, le $P$. mixtum et le $P$. fulvum (Coquillett).

\section{CHEVAUX}

Le $S$. arcticum, le $S$. venustum, le $S$. decorum, le $S$. vittatum et le $S$. luggeri attaquent les chevaux au Canada. Les femelles du $S$. articum piquent principalement sur l'avant-train et la surface inférieure du ventre.

Les femelles du S. venustum et du S. decorum s'alimentent de sang sur presque toutes les parties du corps, mais on croit qu'elles préfèrent 
le poitrail, le côté du cou ou de la tête et les surfaces internes des jambes. Les femelles du S. vittatum sucent surtout le sang des oreilles et causent des dermatoses graves. Toutefois, la simple présence de mouches tournoyant autour de leur tête cause des ennuis aux chevaux.

Les femelles du S. luggeri forment des essaims autour de la tête et piquent notamment les yeux et le chanfrein, ainsi que le côté du cou. Les saignements et les réactions allergiques aux piqûres constituent une caractéristique générale de l'attaque. Les réactions allergiques se présentent sous la forme de grandes papules oedémateuses et de croûtes sanguinolentes sur la surface de la peau. Les chevaux deviennent nerveux et se laissent monter difficilement.

\section{VOLAILLE}

Le $S$. meridionale Riley, le $S$. rugglesi Nicholson \& Mickel et le $S$. aureum Fries sont les principales espèces de parasites de la volaille au Canada. Les attaques varient en intensité dans les différentes régions du pays, selon la proximité des volailles par rapport aux zones de reproduction de ces espèces piqueuses. Les attaques se produisent en général le soir et engendrent une très forte nervosité chez la volaille. Les mouches se nourrissent du sang des crêtes, des caroncules et des parties entourant les yeux et provoquent le saignement et l'apparition de gales. Toutes les espèces énumérées sont des vecteurs de parasites du sang (leucocytozoa spp.), responsables des épidémies qui frappent les dindons, les oies, les canards et les poulets.

\section{MOUTONS}

Le $P$. fulvum, le $P$. esselbaughi Sommerman et le $S$. vittatum sont des espèces connues comme parasites des moutons au Canada. Le $P$. fulvum et le $P$. esselbaughi sont des espèces communes des pâturages alpestres de la Colombie-Britannique. Le $S$. vittatum est actif dans les prairies, particulièrement dans les zones irriguées. Les femelles de ces espèces piquent autour de la tête; il se produit alors chez les moutons une réaction de panique qui les portent à se regrouper pour se protéger. Les pertes que subit le producteur découlent soit des diminutions de poids causées par la réduction du pâturage et aux lésions causées par le rassemblement des moutons en groupes serrés soit des décès des animaux par étouffement.

\section{PORCS}

On sait que le $S$. venustum et le $S$. arcticum s'attaquent aux porcs élevés à l'extérieur. Les attaques de ces mouches rendent les truies stériles et provoquent chez elles l'enflure et l'infection du pis. Les effectifs de reproduction qui n'ont pas été auparavant piqués par les 
mouches noires doivent être protégés pour ne pas être victimes de la salive toxique du $S$. arcticum.

\section{PROTECTION}

\section{PROTECTION DES HUMAINS}

Le harcèlement des humains par les mouches noires peut être réduit de plusieurs façons : le port de vêtements convenables, l'emploi de répulsifs ou en évitant les activités en plein air au cours des périodes où les invasions sont à leur maximum. C'est au cours de la matinée, en fin d'après-midi et au début de la soirée ou encore lorsque le temps est nuageux et que l'orage menace que les attaques des mouches noires sont d'ordinaire plus violentes. Elles sont généralement plus intenses dans les régions boisées qu'en terrain découvert.

\section{Vêtements}

Les habits de couleur claire attirent moins les femelles hématophages. Pour empêcher que les mouches noires ne pénètrent sous les vêtements, il y a lieu de porter des vêtements amples à fermeture éclair, de fermer les manches de chemise et d'introduire le bas des pantalons dans les chaussettes ou les bottes. Il est parfois utile de s'équiper d'un filet fait de matériel à mailles fines pour se protéger la tête et le cou.

\section{Répulsifs}

Appliqués en couche mince et uniforme, les répulsifs assurent une bonne protection contre les mouches noires. Les répulsifs commerciaux contiennent des substances actives telles que le $N, N$-diéthyl-m-toluamide, le 2-éthyl-1,3-hexanediol (6-12), le citronyle et le phtalate diméthyle. Les plus efficaces sont ceux qui contiennent de fortes concentrations de la substance active. Il y a lieu d'appliquer les répulsifs avec soin pour éviter le contact avec les yeux, la bouche et le nez. Il faut toujours lire les instructions figurant sur le contenant avant d'utiliser des répulsifs. Les effets de ces produits sur les vêtements durent longtemps. Les vestons imprégnés de répulsifs sont disponibles sur le marché.

\section{PROTECTION DU BÉTAIL}

On peut protéger les animaux en les élevant le plus loin possible des zones de reproduction des mouches noires, en fournissant des abris pour qu'ils puissent s'y réfugier et éviter les attaques durant les 
périodes d'activité intense, en les traitant avec des insecticides ou des répulsifs et en mettant en oeuvre un programme de traitement au larvicide. Il existe sur le marché plusieurs insecticides et répulsifs qu'il convient d'utiliser selon les directives figurant sur l'étiquette.

\section{Bovins}

Des abris obscurs où les mouches noires ne voleront pas protègent efficacement les bovins contre les attaques de ces insectes. Les abris, qui n'ont pas besoin d'être coûteux pour être efficaces, doivent être non seulement assez grands et solides pour loger le troupeau, mais encore suffisamment obscurs pour empêcher les mouches noires d'y pénétrer. Des bâtiments composés de trois parois solides et d'un toit conviennent d'ordinaire. Un abri de $6 \mathrm{~m}$ de largeur, de $12 \mathrm{~m}$ de profondeur (de l'avant à l'arrière) et de $2,5 \mathrm{~m}$ de hauteur, doté de deux parois latérales solides, d'une paroi arrière et d'un toit s'est révélé satisfaisant pour 20 bovins adultes. Ceux-ci apprennent vite à s'en servir. Dans certaines fermes, des étables ou des dépendances peuvent servir d'abris. Des dispositifs d'épandage automatique de répulsifs et d'insecticides peuvent être installés pour traiter les bovins lorsqu'ils entrent dans l'abri ou en sortent, ce qui en favorise davantage l'utilisation.

Pour être efficaces, les traitements insecticides doivent couvrir la majeure partie du corps de l'animal. Il existe sur le marché plusieurs formules de répulsifs et d'insecticides qui peuvent servir à protéger les bovins contre les attaques des mouches noires. Il ne faut utiliser que les produits recommandés spécialement pour lutter contre ce genre d'insectes et il convient de suivre soigneusement les instructions qui figurent sur l'étiquette. Les restrictions en matière d'utilisation avant l'abattage ou le vêlage doivent être respectées.

On a mis au point un pulvérisateur électrostatique qui permet l'application de répulsifs ou d'insecticides sur les bovins en pâturage. La solution d'insecticide ou de répulsif est projetée sous forme de gouttelettes chargées qui sont attirées vers les poils des animaux. Ce pulvérisateur comporte des avantages : on n'a pas à immobiliser les bovins ni à les enfermer dans l'étable pour traiter toutes les parties de leur corps, on n'a besoin que de petites quantités de solution de pulvérisation et le traitement demande peu de temps.

Des gratte-dos à action chimique, chargés d'insecticides ou de répulsifs, en situation d'utilisation libre ou forcée, assurent une protection contre les mouches noires, et requièrent peu de travail. Des applicateurs à utilisation libre sont placés dans des pâturages ou des enclos et les bovins peuvent s'en servir toutes les fois qu'ils le veulent. Des applicateurs à utilisation forcée sont installés dans les entrées donnant accès à des sources de substances minérales ou d'eau qui sont clôturées. De cette façon, les bovins reçoivent un traitement d'insecticides ou de répulsifs toutes les fois qu'ils entrent dans les enclos ou en sortent. Cette méthode d'application est plus efficace que celle à utilisation libre. 


\section{Chevaux}

Il existe des abris, similaires à ceux des bovins, qui peuvent également assurer aux chevaux une protection temporaire contre les attaques des mouches noires. On peut se procurer sur le marché des répulsifs qu'il convient d'utiliser selon les directives du fabricant. En mettant de la vaseline dans les oreilles des chevaux, on réduit les piqûres des espèces qui s'attaquent surtout aux oreilles.

\section{Volaille}

On peut protéger les volailles des attaques des mouches noires en les gardant à l'intérieur le soir, particulièrement durant les périodes où ces insectes sont les plus actifs.

\section{Moutons et porcs}

Les moutons et les porcs peuvent être temporairement protégés contre les attaques des mouches noires en se réfugiant dans des abris similaires à ceux des bovins.

\section{LUTTE}

\section{PLANIFICATION D'UN PROGRAMME DE LUTTE}

Malgré l'absence de critères définis pour justifier un programme de lutte, il y a lieu d'évaluer les facteurs suivants : la gravité de l'attaque des mouches noires, la proximité des zones de reproduction et le coût de la surveillance et de la lutte. Il faut ensuite communiquer avec des spécialistes, pour qu'ils aident à élaborer un programme de lutte contre les mouches noires qui soit rentable et acceptable sur le plan écologique.

\section{Évaluation de la gravité de l'attaque}

Il convient d'évaluer l'activité et l'abondance des mouches noires adultes, afin de déterminer si des mesures de lutte s'imposent. Plusieurs méthodes peuvent servir à estimer la gravité des attaques par les adultes. Parmi les méthodes courantes, mentionnons l'atterrissage, l'utilisation de pièges gluants et de pièges-mannequin. Comme les espèces réagissent différemment aux diverses méthodes d'évaluation, la méthode utilisée dépendra de l'objectif de l'évaluation et des espèces présentes.

L'atterrissage, qui consiste à placer un bout carré $(30 \times 30 \mathrm{~cm})$ de tissu foncé, de préférence bleu, sur le corps d'une personne et à compter le nombre de mouches qui s'y posent au cours d'un laps de 
temps donné, constitue une méthode utile d'évaluation des populations de mouches noires qui sont attirées vers les humains.

Les pièges gluants, que constituent les boîtes de peinture ou d'autres types d'objets cylindriques, peinturés en bleu et recouverts d'un matériau collant, sont utiles pour attraper les mouches noires dans un secteur donné.

Les pièges-mannequins qui consistent en un cadre de bois ou de métal de forme et de taille similaires à celles d'une vache ou d'un veau et recouvert d'un tissu foncé, ou en une planche de bois recouverte d'une toile de tous côtés sauf au bas, et avec une chambre de collection, sont utiles pour recueillir les mouches noires qui s'attaquent habituellement aux bovins.

Chaque méthode fournit des données quantitatives qui permetent de définir la gravité des attaques et qui peuvent être comparées aux niveaux d'irritation, déterminés en observant le comportement des humains ou des animaux. Ces données peuvent servir également à déterminer le moment approprié pour prendre des mesures de lutte.

\section{Identification des espèces nuisibles et des zones de reproduction}

Des spécimens provenant des dispositifs d'échantillonage des adultes et des larves doivent être conservés dans de l'alcool à friction ou dans de l'antigel à essence. L'emplacement des habitats de reproduction des larves peut être repéré, dans le cas des larves et des pupes, par l'examen des rochers et des plantes dans les parties des ruisseaux et des rivières où le courant est le plus rapide et dans des canaux d'irrigation. Les adultes, les larves et les pupes doivent être conservés, étiquetés en indiquant le lieu et la date de ramassage, puis expédiés à un spécialiste pour être identifiés. Une fois qu'on a déterminé les espèces nuisibles et leurs zones de reproduction et qu'on juge les attaques suffisamment graves, on peut entreprendre un programme de lutte.

\section{Type de programme de lutte}

La destruction des adultes ou des larves au moyen d'insecticides constitue la méthode de lutte la plus courante contre les mouches noires. Un spécialiste devrait superviser ce type de programme. Les programmes de lutte devront être planifiés bien longtemps avant le début de la saison des mouches noires et exécutés de concert avec un entomologiste afin d'assurer l'efficacité des traitements et de causer le moins de dommages à l'environnement. Il importe d'observer toutes les dispositions des lois et des règlements provinciaux portant sur l'utilisation d'insecticides. 
Les mesures de lutte les plus efficaces contre les mouches noires sont celles qui visent la destruction des larves. Étant donné que celles-ci ne se rencontrent que dans les rivières et les cours d'eau et, par conséquent, dans un secteur restreint, leur traitement se révèle très efficace et économique. Dans les zones de reproduction connues, une quantité de larvicide soigneusement dosé et injecté dans l'eau tue les larves et réduit ainsi le nombre de mouches noires.

Ces dernières émergent sur une période prolongée au cours de l'été et il faut plusieurs épandages de larvicide dans une rivière ou un cours d'eau pour réduire sensiblement le nombre de larves. Toutefois, les insecticides, particulièrement l'agent biologique Bacillus thuringiensis israelensis (Bti ou BTH-14), coûtent très cher. Ainsi, un programme de lutte contre les larves doit viser à tuer le plus de larves possible avec un seul épandage de larvicide. Une fois que la relation entre les populations larvaires dans la rivière et la population adulte qui en résulte est établie, on peut mettre en oeuvre un programme de lutte rentable contre les larves des mouches noires, en ayant recours à un dépistage quantitatif du niveau des populations. Ce dépistage du nombre de larves permettra de mieux prévoir les invasions potentielles. Un programme plus intensif de lutte contre les larves peut ensuite être planifié. On peut évaluer les populations larvaires en exposant des substrats artificiels (bouts de corde ou de ruban) auxquels viennent se fixer les larves des mouches noires dans les eaux à débit rapide du cours d'eau à traiter. Lorsque les larves atteignent un nombre suffisant, déterminé en établissant un rapport entre le nombre de larves présentes et la gravité des attaques, comme on l'observe sur les substrats artificiels, on peut décider alors de traiter les zones de reproduction.

\section{Insecticides disponibles}

Les recommandations concernant la lutte contre les mouches noires changent à mesure que des insecticides plus récents et plus efficaces font leur apparition sur le marché. Pour avoir plus de renseignements sur les larvicides actuels, n'hésitez pas à communiquer avec le représentant ou l'agent itinérant du ministère provincial de l'agriculture ou avec Agriculture Canada.

\section{Matériel}

Les larvicides peuvent faire l'objet d'un épandage considérable par hélicoptère sur les zones de reproduction connues le long d'une rivière. Une carte désignant ces zones permet au pilote d'épandre les doses appropriées à chaque endroit. La méthode est rapide, mais coûteuse.

Dans les programmes de moins grande envergure, on épand l'insecticide dilué ou non à partir d'un bateau ou d'un traversier. 
Diverses techniques, notamment l'utilisation de barils, de pulvérisateurs à dos ou d'un arrosoir, peuvent être employées, selon la quantité requise et le type de cours d'eau. Ces méthodes d'épandage sont moins coûteuses que la technique par hélicoptère, mais peuvent prendre du temps, si de nombreuses zones doivent être traitées.

Les petits cours d'eau peuvent être traités au moyen d'un pulvérisateur à dos ou d'un arrosoir.

\section{Calculs des doses de larvicide}

Pour déterminer la quantité d'insecticide nécessaire, il faut connaître le débit du cours d'eau et le dosage requis. Pour calculer le débit de l'eau courante, déterminez la largeur et la profondeur moyenne du cours d'eau en mètres, à un point en amont de la zone à traiter. Mesurez la rapidité de l'écoulement, en mètres par seconde, à différents points avec un moulinet hydrométrique ou en chronométrant la vitesse de passage d'un objet flottant sur une distance précise d'au moins $5 \mathrm{~m}$. L'objet devra être suffisamment lourd pour éviter toute action du vent. Si l'on emploie cette dernière méthode, il faut savoir que la vitesse moyenne est égale à peu près aux deux-tiers de la rapidité de l'écoulement en surface. Le débit du cours d'eau est calculé ensuite de la manière suivante :

Largeur $(\mathrm{m}) \times$ profondeur moyenne $(\mathrm{m}) \times \operatorname{vitesse}(\mathrm{m} / \mathrm{s})=\operatorname{débit}\left(\mathrm{m}^{3} / \mathrm{s}\right)$

On peut mesurer les niveaux d'eau dans les rivières plus importantes au moyen d'une jauge automatique accessible par téléphone. Si la jauge ne donne pas une mesure du débit, consulter le service chargé de son fonctionnement pour savoir comment calculer le débit à partir des relevés du niveau d'eau fournis.

Une fois que le débit est connu et qu'une décision est prise sur le taux d'épandage (dosage), l'utilisation des calculs suivants permet de mesurer la quantité d'insecticide requise :

\section{Quantité requise $=$ débit $\times$ taux d'épandage}

Si un cours d'eau d'un débit de $10 \mathrm{~m}^{3}$ par seconde doit être traité avec du méthoxychlore à $0,3 \mathrm{ppm}$ pendant $15 \mathrm{~min}$, les calculs sont alors les suivants :

$$
10 \mathrm{~m}^{3} \text { d'eau par seconde }=1 \times 10^{7} \mathrm{~g} / \mathrm{s}=900 \times 10^{7} \mathrm{~g} / 15 \mathrm{~min}
$$

La quantité de substance active de méthoxychlore requise pour maintenir une concentration de $0,3 \mathrm{ppm}$, pendant $15 \mathrm{~min}$, dans une rivière dont le débit est de $10 \mathrm{~m} 3 / \mathrm{s}$ est :

$$
\left(900 \times 10^{7} \times 0,3\right) / 10^{6}=2700 \text { g s.a. }
$$

Étant donné qu'il y a $1 \mathrm{~g}$ s.a. par $4,17 \mathrm{~mL}$ de concentré, alors la quantité de concentré requise pour une rivière de ce débit est:

$$
\begin{gathered}
2700 \times 4,17=1125,9 \mathrm{~mL} \text { de concentré } \\
\text { ou } 1125,9 / 1000=11,26 \mathrm{~L}
\end{gathered}
$$


L'insecticide biologique Bacillus thuringiensis israelensis (Bti) est utilisé à différents taux, selon la concentration de la formule et le dosage souhaité. L'étiquette indique la concentration de la formule et les doses recommandées. Par exemple, un produit dont la concentration de la formule est de 1200 unités internationales de toxicité par milligramme est utilisé à des concentrations allant de $1 \mathrm{ppm}$ pendant $15 \mathrm{~min}$ à $25 \mathrm{ppm}$ pendant $1 \mathrm{~min}$. Ainsi, la quantité requise pour traiter un cours d'eau de $10 \mathrm{~m} 3 / \mathrm{s}$ à une concentration de $1 \mathrm{ppm}$ pendant $15 \mathrm{~min}$ sera : $\left(10 \mathrm{~m}^{3} / \mathrm{s} \times 60 \mathrm{~s}=600 \mathrm{~mL} / \mathrm{min}\right.$ de débit $)$ $\times(1 \mathrm{ppm} \times 15 \mathrm{~min})=9000 \mathrm{~mL}=9 \mathrm{~L}$.

\section{Épandage}

L'insecticide est épandu en travers du cours d'eau ou de la rivière durant le laps de temps déterminé par le taux d'épandage (voir les formules de calcul de la concentration de l'insecticide). Le point d'épandage doit être situé suffisamment loin en amont de l'habitat des larves pour que l'insecticide soit convenablement dispersé dans l'eau passant au-dessus de la zone infestée. Par exemple, pour traiter les exutoires des lacs ou des étangs fréquentés par des castors, il faut répandre l'insecticide dans le lac ou l'étang, suffisamment loin de l'exutoire de façon à ce que l'insecticide puisse être dispersé jusqu'au fond avant d'arriver dans l'exutoire. Plus près de l'exutoire, l'insecticide peut être épandu au fond ou à proximité du fond au moyen d'un tuyau installé sur un bateau. Dans les grandes rivières, comme l'Athabasca ou la Saskatchewan, il faudra choisir une méthode capable d'assurer une dispersion et une dissolution complètes ainsi qu'une concentration uniforme. Il importe de savoir sur quelle distance l'insecticide est efficace (autrement dit sur quelle distance $80 \%$ des larves ou plus sont détruites) afin de déterminer le nombre de points d'épandage de l'insecticide dans la zone de traitement. Il faut également tenir compte de l'intervalle entre les traitements. Si un premier traitement ne réduit pas sensiblement la population larvaire, d'autres traitements seront peut-être nécessaires. En outre, les espèces de mouches noires qui produisent plus d'une génération par année devront faire l'objet de plusieurs traitements durant l'année.

Lorsqu'on envisage de mettre en oeuvre un programme de lutte contre les larves, il faut consulter des spécialistes, parce qu'ils connaissent les techniques nécessaires à l'élaboration d'un programme efficace. Il convient également d'obtenir une autorisation du ministère provincial de l'environnement, toutes les fois que des insecticides sont épandus dans l'eau courante.

\section{DESTRUCTION DES ADULTES}

Des pulvérisations aériennes bien exécutées peuvent réduire temporairement les attaques des mouches noires adultes. Une pulvérisation aérienne, sous forme de brouillard fin ou d'aérosol, 
consiste en de très fines gouttelettes d'une solution d'insecticide qui sont dispersées par les mouvements de l'air. Les gouttelettes entrent en contact avec les mouches noires adultes qui sont en vol ou au repos. Toutefois, étant donné que les pulvérisations aériennes ne produisent pas de dépôts de résidus d'insecticide efficaces, le soulagement est temporaire et il faudra peut-être reprendre les traitements chaque jour, tant que les adultes constituent une source de problèmes.

La pulvérisation de la végétation dans les fermes, autour des camps de bûcherons, des terrains de camping et des aménagements récréatifs, au moyen d'insecticides dotés de propriétés résiduelles, réduira temporairement les attaques des mouches noires. L'insecticide peut être épandu sous forme de brouillard fin ou de fines gouttelettes.

\section{Insecticides disponibles}

Les recommandations concernant l'utilisation d'insecticides pour lutter contre les mouches noires changent à mesure que des insecticides plus récents et plus efficaces font leur apparition sur le marché. Pour avoir plus de renseignements sur les insecticides capables de détruire les mouches noires, adultes, n'hésitez pas à communiquer avec le représentant ou l'agent itinérant du ministère provincial de l'agriculture ou avec Agriculture Canada.

\section{Matériel}

Un pulvérisateur à air comprimé, doté d'une buse de pulvérisation de gouttelettes fines ou d'un générateur d'aérosols, peut disperser suffisamment l'insecticide pour réduire temporairement le nombre de mouches noires adultes dans des zones restreintes. Les générateurs d'aérosols peuvent épandre jusqu'à $454 \mathrm{~L} / \mathrm{h}$ du mélange insecticide sur de grandes superficies. Il se produit un nuage de gouttelettes très fines qui sera dispersé par le vent.

\section{Épandage}

Le pulvérisateur à air comprimé devra être rempli de l'insecticide choisi aux trois quarts et l'air sera comprimé au moyen de la pompe à main. La pulvérisation peut durer jusqu'à ce que la pression totale tombe à un niveau où le traitement devient inégal. La pression devra alors être de nouveau augmentée à l'aide de la pompe à main.

Lorsqu'on utilise un nébuliseur (générateur d'aérosols à thermo-déclenchement), il faudra épandre l'insecticide juste avant le coucher ou le lever du soleil, au moment où les mouches noires adultes sont très actives. La vitesse du vent qui convient le mieux aux épandages au sol est de moins de $10 \mathrm{~km} / \mathrm{h}$. Il convient de déplacer l'appareil à une vitesse de 5 à $6 \mathrm{~km} / \mathrm{h}$ en lignes parallèles, perpendiculairement à la direction du vent, et laisser 200 à $350 \mathrm{~m}$ 
entre deux lignes. Si on utilise un générateur d'aérosols dans les zones où il y a risque d'incendie, il y a lieu d'épandre l'insecticide sous forme d'émulsions aqueuses plutôt que de solutions huileuses.

\section{ÉVALUATIONS ETT RAPPORTS}

Il convient d'évaluer l'efficacité des mesures de lutte dans les $48 \mathrm{~h}$ qui suivent les épandages d'insecticides. Une partie importante du programme consiste à réunir de la documentation sur les méthodes de lutte et surveiller les résultats. Les comptes rendus concernant le nombre de larves et d'adultes durant la saison des mouches noires fournissent des renseignements importants qui permettent de poursuivre ou de modifier un programme de lutte. Un rapport récapitulatif ou annuel rédigé à la fin de la saison aidera dans une large mesure à planifier les programmes futurs. Le rapport devra comprendre des données sur les tendances d'évolution des populations, les calendriers de traitement au moyen d'insecticides, les quantités d'insecticides utilisées, les zones couvertes, les méthodes de traitement, les coûts et tous les autres faits pertinents. Des prévisions peuvent également être établies en fonction de l'information consignée. 
TABLE DE CONVERSIONS

\section{Longueur}

pieds

verges

milles

\section{Surface}

pouces carrés

pieds carrés $\left(p i^{2}\right)$

verges carrées $\left(v^{2}\right)$

acres

acres

\section{Volume}

onces liquides

onces liquides (U.S.)

chopines

pintes

gallons

gallons (U.S.)

pieds cubes

pouces cubes

\section{pieds cubes}

verges cubes

\section{Poids}

onces

livres

tonne courte

\section{Vitesse}

milles/heure (mph) pieds/seconde

\section{Agriculture}

\section{onces/acre}

livres/acre

chopines/acre

gallons/acre

gallons/acre (U.S.)

plants/acre

tonnes/acre

onces liquides/acre

\section{température}

degrés

\section{Pression}

livres/pouce carré

$\times 0,84$

$\times 4047$

$\times 0,405$

$\times 28,41$

$\times 29,57$

$\times 0,57$

$\times 1,14$

$\times 4,55$

$\times 3,79$

$\times 28,32$

$\times 16,39$

$$
\times 0,03
$$$$
\times 0,77
$$

$\times 0,45$$$
\times 1,61
$$$$
\times 30,48
$$

$$
\begin{array}{ll}
\times & 70,05 \\
\times & 1,12 \\
\times & 1,40 \\
\times & 11,23 \\
\times & 9,35
\end{array}
$$

$$
\begin{aligned}
\text { centimètres } & (\mathrm{cm}) \\
\text { mètres } & (\mathrm{m}) \\
\text { kilomètres } & (\mathrm{km})
\end{aligned}
$$

$\begin{aligned} \text { centimètres carrés } & \left(\left(\mathrm{cm}^{2}\right)\right. \\ \text { mètres carrés } & \left(\mathrm{m}^{2}\right) \\ \text { mètres carrés } & \left(\mathrm{m}^{2}\right) \\ \text { mètres carrés } & \left(\mathrm{m}^{2}\right) \\ \text { hectares } & (\mathrm{ha})\end{aligned}$

millilitres $(\mathrm{mL})$ millilitres $(\mathrm{mL})$

litres (L)

litres (L)

litres (L)

litres (L)

litres (L)

centimètres cubes $\left(\mathrm{cm}^{3}, \mathrm{cc}\right)$, millilitres $(\mathrm{mL})$ metres cubes $\left(\mathrm{m}^{3}\right)$ mètres cubes $\mathrm{m}^{3}$ )

grammes (g) kilogrammes (kg) tonne forte

kilomètres/heure $(\mathrm{km} / \mathrm{h})$ mètres/seconde $(\mathrm{m} / \mathrm{s})$

grammes/hectare (g/ha) kilogrammes/hectare (kg/ha) litres/hectare (L/ha)

litres/hectare (L/ha)

litres/hectare (L/ha) plants/hectare

tonnes/hectare millilitres/hectare

degrés

kilopascal

\section{Puissance}

chevaux (CV)

Watt/kilowatt 


